\title{
A new library-search algorithm for mixture analysis using DART-MS
}

Arun S. Moorthy* and Edward Sisco

National Institute of Standards and Technology, Gaithersburg, MD, 20899 USA

*DARTdata@nist.gov

Abstract: This manuscript introduces a new library-search algorithm for identifying components of a mixture using in-source collision-induced dissociation (is-CID) mass spectra. The two-stage search, titled the Inverted Library-Search Algorithm (ILSA), identifies potential components in a mixture by first searching its low fragmentation mass spectrum for target peaks, assuming these peaks are protonated molecules, and then scoring each target peak with possible library matches using one of two schemes. Utility of the ILSA is demonstrated through several example searches of model mixtures of acetyl fentanyl, benzyl fentanyl, amphetamine and methamphetamine searched against a small library of select compounds and the NIST DART-MS Forensics library. Discussion of the search results and several open areas of research to further extend the method are provided. A prototype implementation of the ILSA is available at https://github.com/asm3-nist/DART-MS-DST.

\section{Keywords: Algorithms, DART-MS, Library Searching, Mass Spectrometry}

\section{Introduction}

Direct Analysis in Real Time Mass Spectrometry (DART-MS) has been a critical technology in the highthroughput analysis of chemicals under ambient conditions [1], with several important applications being demonstrated in the recent past (See $[2,3]$ and references therein). Broadly speaking, DART-MS employs a heated stream of metastable gas molecules (typically helium) to both desorb and, through a cascade of steps, ionize a sample. While rapid, because there is no chromatography, resulting mass spectra are often complex as the spectral signatures for all ionizable compounds in mixture are observed simultaneously. DART is considered a soft ionization technique and therefore mainly intact molecular ions (protonated molecules) are produced. While useful for determining potential molecular formulae, in-source collisioninduced dissociation (is-CID) can also be employed to obtain one or more fragmentation spectra that can be used to further assist in structure elucidation and compound identification. These multiple is-CID spectra represent the sample measured with various levels of fragmentation as controlled by settings specific to the employed mass spectrometer. An example set of is-CID spectra for a single sample collected using a JEOL AccuTOF (Peabody, MA, USA) are provided in Figure 1.

The National Institute of Standards and Technology (NIST) has a long history of collecting mass spectra for reference compounds, producing high-quality reference mass spectral databases (or libraries) for electron ionization mass spectrometry (EI-MS) and electrospray ionization tandem mass spectrometry (ESI-MS/MS)[4]. These databases of reference spectra can be utilized in several ways, but possibly of most importance is their use in compound identification. An analyst can query the spectrum of an analyte against reference libraries; the mass spectrum of the analyte is compared sequentially to the reference spectra and the most similar entries in the database are returned to the user as an ordered "hit list" (sometimes written as "hits list" or "hitlist"). This process is commonly referred to as mass spectral library searching. An analyst can then decide whether their analyte is one of the compounds in the returned hit list. 

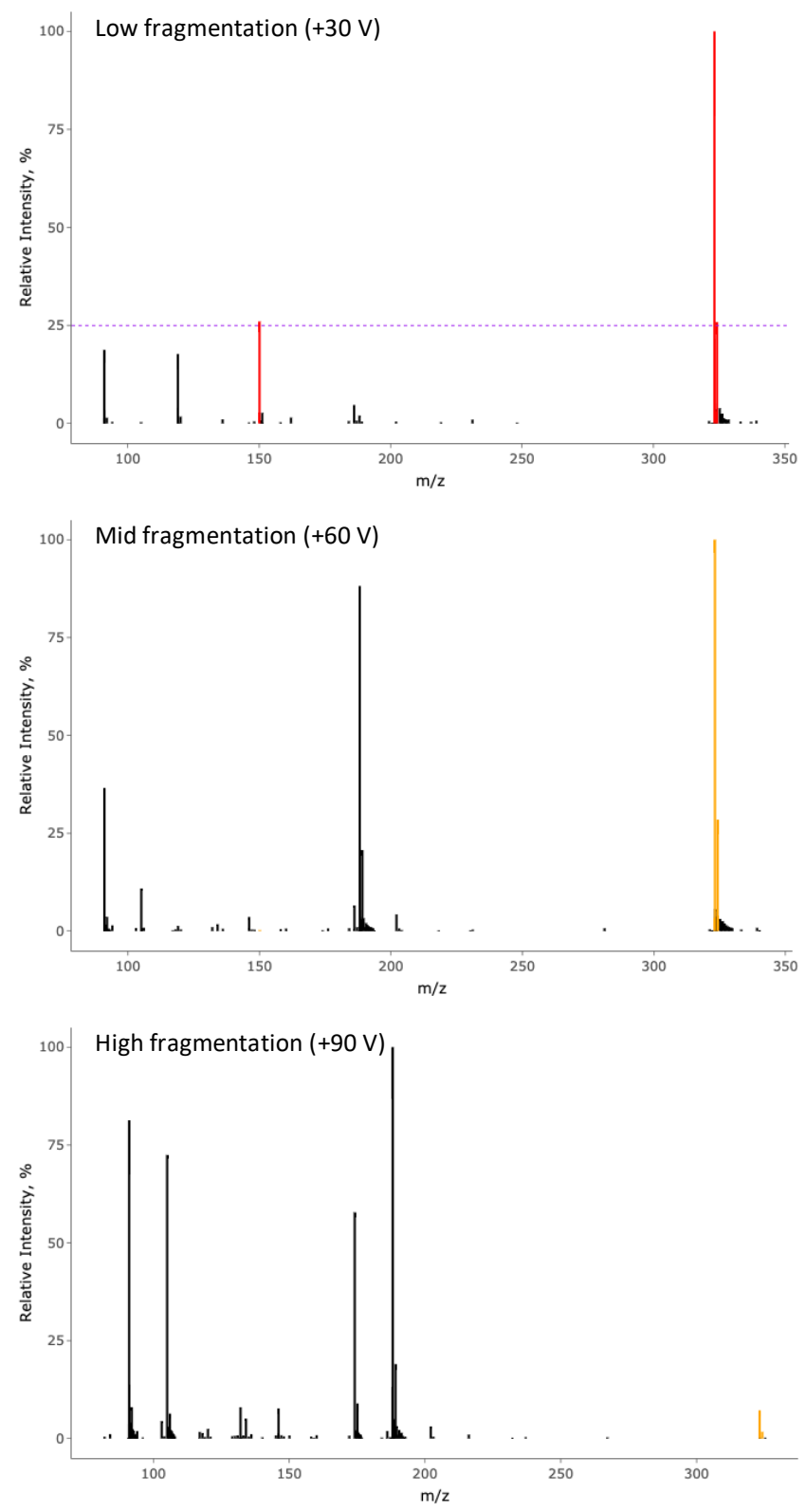

Figure 1: In-source collision-induced dissociation (is-CID) mass spectra of Mixture 3 (Table 1) measured using a JEOL AccuTOF (Peabody, MA, USA) mass spectrometer, with orifice 1 energy settings of $+30 \mathrm{~V},+60 \mathrm{~V}$ and $+90 \mathrm{~V}$. Potential targets with a relative intensity greater than $25 \%$ (i.e., with peak intensities above dashed horizontal line) in $+30 \mathrm{~V}$ spectrum are highlighted in red. The peaks corresponding to the target(s) identified in the low fragmentation spectrum are highlighted in orange in the higher fragmentation spectra. This figure is further discussed in Example 1 of Section 3.1.

Recently, NIST has released an updated DART-MS Forensics mass spectral library [5,6]. The library contains multiple is-CID mass spectra of over 650 pure compounds of interest to the forensics community (e.g., seized drugs, cutting agents, etc.). The spectra in this library can be viewed, interpreted, and even searched against using standard mass spectral library search tools like NIST MS Search [7]. However, there are two major drawbacks to using standards search tools: (1) spectra are searched individually, requiring an analyst to later manually reconcile the search results for each of the multiple is-CID spectra for the same 
analyte, and (2) DART-MS is rarely preceded by chromatography and so the query spectrum is usually of a mixture and not pure compound.

The objective of this manuscript is to present an algorithmic approach for determining presumptive identifications of mixture components from a DART-MS experiment using a pure compound library. The method leverages the multilevel information contained in centroided is-CID spectra to first match possible library entries to mixture components targeted in the measurement with least fragmentation, and then assign classification indices, or scores, based on the spectral similarity of all centroided is-CID spectra of the sample. Unlike the more traditional search paradigm, where scores are computed to reflect how well peaks in the query (mixture) mass spectra are explained by matching peaks in the library (pure compound) spectra, the scoring in this algorithm reflects how well peaks in the library spectra are explained by matching peaks in the query. We refer to this new approach as the inverted library-search algorithm (ILSA). Utility of the ILSA is demonstrated through several example searches. In particular, the searches are applied to the challenge of identifying components of controlled mixtures containing up to four illicit drugs present at equal concentrations. The drugs considered in the controlled mixtures are acetyl fentanyl, benzyl fentanyl, amphetamine and methamphetamine. These drugs were selected as representatives of two important scenarios: (1) compounds with identical protonated molecules but distinct fragment ions (acetyl fentanyl and benzyl fentanyl), and (2) compounds with unique protonated molecules but similar fragment ions (amphetamine and methamphetamine).

The manuscript is structured as follows. Section 2.1 provides a comprehensive description of the ILSA, specifying assumptions and remarking on known limitations. Description of a prototype software implementation and the experimental design employed to test the algorithm follows in Section 2.2. Discussion of selected demonstrative examples are included as Section 3.1 and a discussion of the full search results as well as a discussion of potential extensions and future work comprises Section 3.2.

\section{Materials and Methods}

\subsection{Algorithm Details}

To most intuitively present the inverted library-search algorithm (ILSA), we describe its underlying assumptions, implementation details, and relevant remarks in a comprehensive manner.

An extracted is-CID mass spectrum is a list of coupled mass-to-charge ratios $(\mathrm{m} / \mathrm{z})$ and normalized intensities (\%) of the ions measured in a sample. Let $x_{M}^{E}$ denote the mass spectrum of a mixture $M$ measured with fragmentation condition $E$. The mechanisms that impact fragmentation conditions will vary depending on the particular mass spectrometer employed. In this manuscript, we employ a JEOL AccuTOF (Peabody, MA, USA) mass spectrometer where the level of fragmentation increases with increasing orifice 1 energy. For this instrument, typical orifice 1 energy values are $\pm 30 \mathrm{~V}, \pm 60 \mathrm{~V}$, and $\pm 90 \mathrm{~V}$, resulting in low, mid, and high fragmentation spectra. Let $l_{i}^{E}$ denote a reference mass spectrum of a pure compound $i$ measured with fragmentation condition (orifice 1 energy) $E$ as previously described for mixtures.

Assumption 1: The component molecules contained in a mixture will each present a protonated molecule in

$x_{M}^{30}$ (or the low fragmentation spectrum considered in the experiment) and the relative intensity of these protonated molecules will be greater than a threshold relative intensity denoted $\tau_{R I}$. 
Algorithm Steps:

1) Review the $x_{M}^{30}$ for potential protonated molecules which we can refer to as targets.

i. Identify target $m / z$ in $x_{M}^{30}$ by locating peaks with relative intensities greater than $\tau_{R I}$ (e.g., $25 \%$ ).

ii. Record the target $m / z$ values as set $\boldsymbol{T}$.

Remark 1: While assumption 1 is likely to hold for most mixture components, there are several reasons why certain components may not appear in a mixture spectrum with relative intensity greater than $\tau_{R I}$ :

i) The component does not normally produce a substantial protonated molecule peak.

ii) The component is at too low of a concentration to be detected.

iii) Competitive ionization within the mixture prohibits sufficient ionization of the component.

If a mixture component does not present a protonated molecule with relative intensity greater than $\tau_{R I}$ it will not be identified as a target in algorithm step 1. An alternative strategy that has been explored is assuming identified targets are base peak $m / z$ values rather than protonated molecules, as will be discussed in the worked examples of Section 3.1. With this assumption, all mentions of protonated molecules in the algorithm can be replaced with base peak. Note that for many molecules, the protonated molecule is the base peak and so the algorithm will perform identically. We should also note that if a mixture is analyzed in negative ion mode, we would see deprotonated molecules in the spectrum rather than protonated molecules.

Assumption 2: Reference mass spectra of the component molecules contained in the mixture are available in a searchable database ${ }^{1}$. The difference between protonated molecule $m / z$ values of database entries and the targets obtained from $x_{M}^{30}$ in Algorithm Step 1 is accurate to a known instrument resolution $\left( \pm \epsilon_{0}\right)$. The difference in $m / z$ values of the peaks in the reference spectra and corresponding peaks in the mixture spectrum will be within a defined $m / z$ resolution interval $\left( \pm \epsilon_{1}\right)$. In most cases, it may be further assumed that $\epsilon_{1}$ is a constant and $\epsilon_{1}=\epsilon_{0}$.

Algorithm Steps:

2) For each entry $t$ in set $\boldsymbol{T}$ :

i. Search the database for entries with a protonated molecule at $m / z$ value within $\pm \epsilon_{0}$ units of target $t$.

ii. Record the index of these database entries as set $\boldsymbol{r}$.

Remark 2: If a component of mixture $M$ does not have a representative spectrum present in the reference library, it will not be directly identifiable through the ILSA. It is important that reference databases are continually developed and improved upon. Additionally, indirect search procedures, such as the Hybrid Similarity Search employed in both electron ionization [8] and electrospray ionization $[9,10]$ mass spectral library searching, may help mitigate the limitations of incomplete libraries and may be a fruitful avenue for future work. Assuming that $\epsilon_{1}$ is constant and $\epsilon_{1}=\epsilon_{0}$ should be reasonable for small molecules, however, we might expect that mass spectra of larger molecules may suffer from mass drift and thus $\epsilon_{1}$ will not be

\footnotetext{
${ }^{1}$ A searchable database will include mass spectra for reference compounds as well as important metadata such as known protonated molecule $m / z$ value, observed protonated molecule $m / z$ value, observed base peak $m / z$ value, etc.
} 
constant. Additionally, if both query and library spectra are not measured using high-resolution mass spectrometry, $\epsilon_{0}$ and $\epsilon_{1}$ must be decoupled. In general, one may improve accuracy for any search condition by decoupling $\epsilon_{0}$ and $\epsilon_{1}$ and optimizing values for particular use-cases.

Assumption 3: If a molecule from the reference database is a component of the mixture:

(a) peaks from its reference (database) mass spectra are likely to be represented within the mass spectra of the mixture, with likelihood of appearance being a function of the relative intensities of the peak (i.e., the reference spectrum base peak is more likely to appear in the mixture spectrum than a low intensity peak), and

(b) the $m / z$ difference (drift or bias) between the reference spectra peaks and the matched peaks in the mixture mass spectra will be consistent.

Algorithm Steps:

3) For each entry $i$ in set $r$ :

i. Identify the peaks in $l_{i}^{30}$ with $m / z$ less than or equal to the protonated molecule $m / z+\epsilon_{0}$ and with corresponding peaks in $x_{M}^{30}$ within $\pm \epsilon_{1}$ units and denote this set $\boldsymbol{a}_{30} \cdot{ }^{2}$

ii. Compute a spectral similarity score (see Remark 4) for $l_{i}^{30}$ based on the peaks identified in set $\boldsymbol{a}_{\mathbf{3 0}}$. Refer to this value as spectral similarity with orifice energy $+30 \mathrm{~V}$ and denote it as $\alpha_{30}$. Repeat process to compute spectral similarity scores for measurements with other orifice energies (e.g., $\alpha_{60}$ and $\alpha_{90}$ ).

iii. Compute the weighted average spectral similarity $(\tilde{\alpha})$

$$
\tilde{\alpha}=\sum_{e \in E} \omega_{e}^{\alpha} \alpha_{e}
$$

where $E=\{30,60,90\}$ and $\omega_{e}^{\alpha}$ are tunable weights between 0 and 1 such that $\sum_{e \in E} \omega_{e}^{\alpha}=1$.

iv. For each peak identified in set $\boldsymbol{a}_{\mathbf{3 0}}$, determine the absolute difference between each $\mathrm{m} / \mathrm{z}$ value in $l_{i}^{30}$ and its closest $m / z$ value in $x_{M}^{30}$. Refer to this set of absolute differences as $\boldsymbol{b}_{\mathbf{3 0}}$. Compute the standard deviation of entries in set $\boldsymbol{b}_{\mathbf{3 0}}$. Refer to this value as the mass bias with orifice energy $+30 \mathrm{~V}$ and denote it as $\beta_{30}$. Repeat process for $\beta_{60}$ and $\beta_{90}$.

v. Compute the weighted average mass bias $(\tilde{\beta})$

$$
\tilde{\beta}=\sum_{e \in E} \omega_{e}^{\beta} \beta_{e}
$$

\footnotetext{
${ }^{2}$ An upper limit on $m / z$ values is employed to prevent computations including obvious noise and/or dimer peaks.
} 
where $E=\{30,60,90\}$ and $\omega_{e}^{\beta}$ are tunable weights between 0 and 1 such that $\sum_{e \in E} \omega_{e}^{\beta}=1$.

vi. Compute the absolute mass difference $(\gamma)$ between the target $m / z$ value $t$ and the known protonated molecule $m / z$ value of $l_{i}$. Note that this is a single value and is only dependent on the target $m / z$ value identified in $x_{M}^{30}$.

vii. Compute a classification index $\left(\theta_{i}\right)$ for the library entry $l_{i}$ as

$$
\theta_{i}=\tilde{\alpha} \cdot(1-\tilde{\beta}) \cdot(1-\gamma)
$$

The value $\theta_{i}$ can then be used for classification decisions.

Remark 3: Assumption 3 relies on the is-CID spectra of the mixture and reference pure compounds being taken under similar conditions. Characterizing the range of conditions for which one can assume peaks in is-CID spectra are sufficiently reproducible between mixtures and pure compounds is necessary future work.

Remark 4: Algorithm step 3ii introduces the concept of a spectral similarity score denoted $\alpha_{E}$ where $E \in$ $\{30,60,90\}$. The objective of a spectral similarity score is to provide a numerical value that meaningfully characterizes the similarity between a pair of mass spectra. There are several measures of spectral similarity readily available for comparing spectra of pure compounds as described in $[11,12]$ and references therein. In this manuscript we consider two scoring methods that estimate the similarity between a pure compound spectrum $\left(l_{i}^{E}\right)$ and a mixture spectrum $\left(x_{M}^{E}\right)$ : (1) fraction of relative intensity of peaks in $l_{i}^{E}$ with matching peaks within $\pm \epsilon_{1}$ in $x_{M}^{E}$, and (2) the cosine similarity between the vectors of relative intensity for peaks in $l_{i}^{E}$ with the closest peaks within $\pm \epsilon_{1}$ in $x_{M}^{E}$. Both scoring methods return real numbered values between 0 and 1 inclusive. We refer to the first score as the "fraction of library peak intensity explained" (FPIE) and the second as a "reverse match factor" (RevMF) based on its historical application in mass spectral library searching. Example calculations of both scores are provided as Appendix A.

The classification index calculated in equation (3), and its dependent parameters computed using assumed weights in algorithm steps 3ii-3vi, is a simple first approximation of likelihood based on mass spectral similarity scores and $m / z$ differences between matching peaks. It is possible that optimizing the weights used within equations (1) and (2) or developing novel similarity scores that more meaningfully measure spectral similarity between pure compounds and mixtures will produce improved approximations of likelihood and subsequently improve algorithm effectiveness.

Summary of inverted library-search algorithm: The ILSA can be summarized as a two-stage procedure beginning with a target identification stage (steps 1 and 2) and ending with a scoring stage (step 3). The target identification determines matches from the reference library that may be components of the analyte mixture and scoring computes an index for classification/decision-making about each library match. For target identification, our initial implementation uses a relative intensity threshold and assumes that peaks with relative intensity above the threshold are protonated molecules (or base peaks) of library compounds. 
For scoring, we compute classification indices that combined one of two spectral similarity scores (FPIE or RevMF), the weighted mass bias between corresponding peaks and the absolute mass difference between the identified target $\mathrm{m} / \mathrm{z}$ and the protonated molecule. A graphical summary of the ILSA is provided as Figure 2. Overall improvements with future implementations of the ILSA will be the result of improved target identification and scoring of library matches.

Application of the ILSA for pure compound analysis: While exploring the effectiveness of pure compound analysis using is-CID is outside the scope of this manuscript, we note that the only special requirement for pure compound analysis is that the target relative intensity threshold $\tau_{R I}$ is set to $100 \%$ in algorithm step 1 such that only a single peak is targeted for further analysis. All other algorithm steps can proceed as described.

Application of the ILSA for "mixed resolution" spectral searching: Though not explicitly stated, the discussion thus far has assumed that both query and library mass spectra are measured with high-resolution instrumentation. A detailed discussion of the efficacy of the ILSA when one or both query and library mass spectra are integer resolution is outside of the scope of this manuscript. However, we will briefly summarize our current implementation for this use-case. If both query and library spectra are integer resolution, we set $\epsilon_{0}=1$, allowing a wider range of target $m / z$ values to be identified and set $\epsilon_{1}=0$ to ensure only peaks with exact $m / z$ value matches are used in similarity scoring. If only one of the query or library spectra are integer resolution, the high-resolution spectra is approximated by an integer resolution mass spectrum where $m / z$ values are rounded and peaks with the same $m / z$ are consolidated by summing intensity. The search can then proceed as though both query and library spectra are integer-resolution.

Application of the ILSA for other non-chromatographic MS techniques: While not evaluated specifically, we believe the outlined approach is applicable with other ambient ionization sources and/or direct infusion sources given appropriate pure compound libraries and algorithm modifications. 


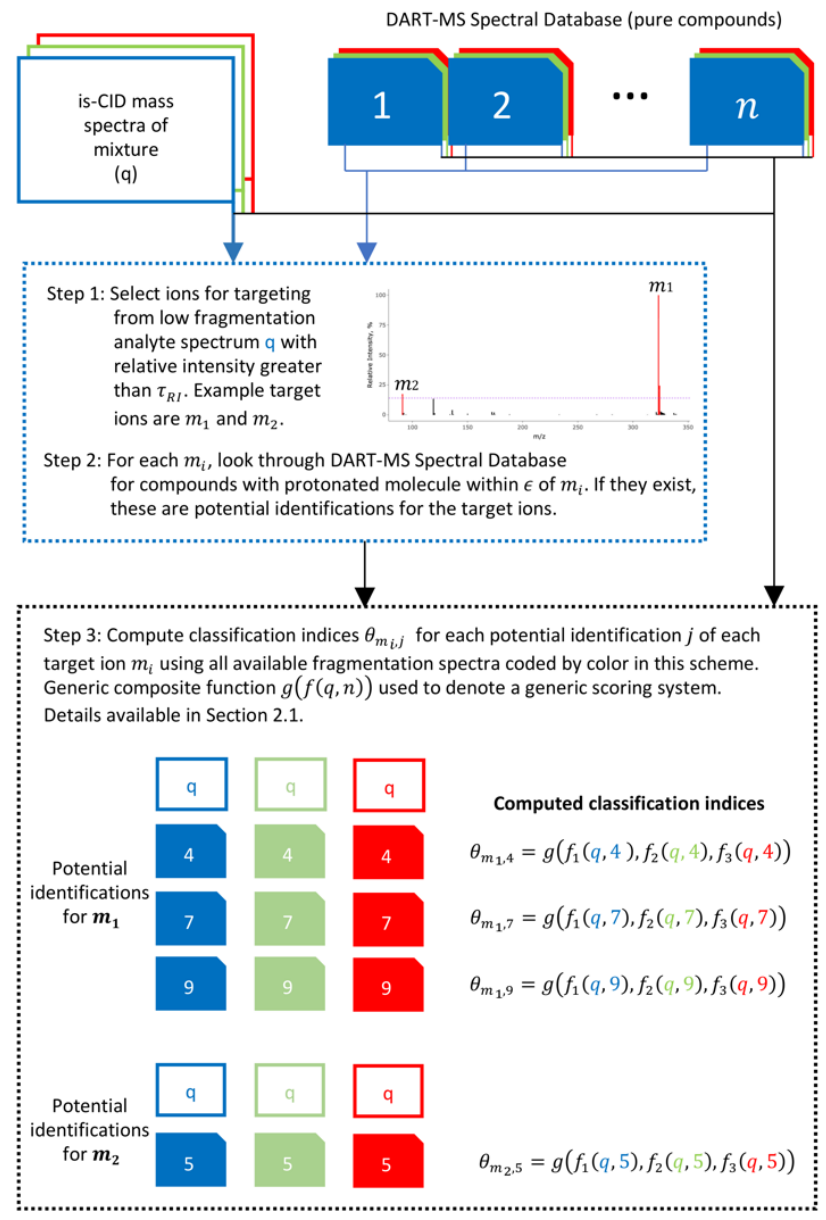

Figure 2: Summary of the Inverted library-search algorithm (ILSA). Color coding is as follows: blue - low fragmentation spectrum, green - mid fragmentation spectrum, red - high fragmentation spectrum. Low fragmentation (blue) spectra are used in target identification (steps 1 and 2), whereas all fragmentation spectra are involved with scoring (step 3). 


\subsection{Software Implementation and Test Data}

The ILSA for mixture and pure compound analysis is implemented within the new NIST DART-MS Database Search Tool (DST). The tool is written in R[13] and follows a Shiny[14] framework. The source code is available at https://github.com/asm3-nist/DART-MS-DST. The search tool requires reference libraries in R data table (.RDS) format and search spectra as tab-delineated text files (.txt). Reference libraries can be generated in .RDS format from mass spectra and metadata using the NIST DART-MS Database Builder Program available at https://github.com/asm3-nist/DART-MS-DBB. The example spectra and libraries discussed in this manuscript are available with the NIST DART-MS DST download.

To demonstrate the utility of the ILSA for mixture analysis, we created 11 mixtures composed of 2 to 4 drugs in equal concentration: acetyl fentanyl, benzyl fentanyl, amphetamine and methamphetamine. A summary of the components included in each of the 11 mixtures is provided as Table 1. Each mixture was measured using DART-MS at orifice 1 energies of $+30 \mathrm{~V},+60 \mathrm{~V}$, and $+90 \mathrm{~V}$ to generate is-CID query mass spectra. The mixtures were searched against a select library of measurements for pure samples of acetyl fentanyl, benzyl fentanyl, amphetamine and methamphetamine, collected at the same time as the mixtures, to confirm algorithm functionality, as well as the NIST DART-MS Forensics Database $[5,6]$ to investigate more general utility.

Mass spectra were collected using a JEOL AccuTOF mass spectrometer (JEOL) coupled with a DARTSVP ion source (IonSense, Saugus, MA, USA). Relevant DART parameters include operation in positive ionization mode with ultra-pure helium as the source gas and a gas temperature of $400{ }^{\circ} \mathrm{C}$. The mass spectrometer was also operated in positive ionization mode using an orifice 2 and ring lens voltage of +5 $\mathrm{V}$, and a $+600 \mathrm{~V}$ rf guide voltage. Parameter switching was used to cycle through $+30 \mathrm{~V},+60 \mathrm{~V}$, and +90 $\mathrm{V}$ orifice 1 voltages at a rate of $0.2 \mathrm{~s} /$ cycle. Mass spectra were collected from $\mathrm{m} / \mathrm{z} 80$ to $\mathrm{m} / \mathrm{z} 800$ at 2 scan/s. Each drug mixture was made, individually, using $1 \mathrm{mg} / \mathrm{mL}$ solutions of the pure drugs purchased from Cayman Chemical (Ann Arbor, MI, USA). For each mixture a total volume of $200 \mu \mathrm{L}$ was made, with drugs present at a concentration of $0.25 \mathrm{mg} / \mathrm{mL}$ in methanol. Samples were introduced to the DART gas stream via dipped glass microcapillaries. This process was completed three times for each mixture and the composite mass spectra from the replicates were extracted. Query spectra were background subtracted against a spectrum of methanol obtained in the same run and then centroided using Mass Center (JEOL).

Table 1: Summary of mixture compositions considered in this study. Components marked $(\bullet)$ are included in the mixture, whereas unmarked components are not included in the particular mixture.

\begin{tabular}{lccccccccccc}
\hline Mixture & 1 & 2 & 3 & 4 & 5 & 6 & 7 & 8 & 9 & 10 & 11 \\
\hline Acetyl fentanyl & $\bullet$ & $\bullet$ & $\bullet$ & & & & $\bullet$ & $\bullet$ & $\bullet$ & & $\bullet$ \\
Benzyl fentanyl & $\bullet$ & & & $\bullet$ & $\bullet$ & & $\bullet$ & $\bullet$ & & $\bullet$ & $\bullet$ \\
Amphetamine & & $\bullet$ & & $\bullet$ & & $\bullet$ & $\bullet$ & & $\bullet$ & $\bullet$ & $\bullet$ \\
Methamphetamine & & & $\bullet$ & & $\bullet$ & $\bullet$ & & $\bullet$ & $\bullet$ & $\bullet$ & $\bullet$ \\
\hline
\end{tabular}




\section{Results and Discussion}

\subsection{Example Searches}

Consider the low fragmentation $(+30 \mathrm{~V})$ is-CID mass spectrum shown in Figure 1. This is a measurement of Mixture 3 from Table 1 and includes acetyl fentanyl and methamphetamine. Using a relative intensity threshold $\tau_{R I}=25 \%, 3$ targets are identified (step 1): (1) $\mathrm{m} / z 323.214$ with relative intensity $100 \%$, (2) $\mathrm{m} / \mathrm{z} 150.130$ with relative intensity $26.0 \%$, and (3) $\mathrm{m} / \mathrm{z} 324.217$ with relative intensity $25.8 \%$.

For target 1, both acetyl fentanyl and benzyl fentanyl are compounds in the select library with protonated molecules of $m / z 323.214 \pm 0.005$ (step 2). For the reference acetyl fentanyl, the spectral similarity scores of the $+30 \mathrm{~V},+60 \mathrm{~V}$ and $+90 \mathrm{~V}$ spectra, using FPIE, are 0.988, 0.990 and 0.966 , respectively, resulting in a weighted average spectral similarity score (Equation 1 ) of 0.981 with equal weights $(0 . \overline{3})$ for all three isCID mass spectra (steps 3i-iii). The weighted mass bias and absolute mass difference (Algorithm steps 3ivvi) minimally impact the computation of the classification index of 0.979 (Algorithm step 3vii). If we use RevMF for scoring spectral similarity, the scores of the $+30 \mathrm{~V},+60 \mathrm{~V}$ and $+90 \mathrm{~V}$ spectra are 1.000, 0.998 and 0.987 , respectively, resulting in a weighted average spectral similarity score of 0.995 with equal weighting. The weighted mass bias and absolute mass difference minimally impact the final computed classification index of 0.992 . For the reference benzyl fentanyl, which produces different fragmentation spectra than acetyl fentanyl, the spectral similarity scores of the $+30 \mathrm{~V},+60 \mathrm{~V}$ and $+90 \mathrm{~V}$ spectra using FPIE are $0.921,0.496$, and 0.516 , respectively, resulting in a weighted average spectral similarity score of 0.644. Using RevMF, the spectral similarity scores are $0.983,0.786$, and 0.207 , resulting in a weighted average spectral similarity score of 0.659 . The weighted mass bias and absolute mass difference minimally impact the computed classification indices of 0.642 and 0.657 using FPIE and RevMF, respectively. For target 2, only methamphetamine from the Select library matches with protonated molecule within $\pm 0.005 \mathrm{~m} / \mathrm{z}$ of $\mathrm{m} / \mathrm{z} 150.130$, and the computed classification indices are 0.895 and 0.969 for scoring using FPIE and RevMF, respectively. Target 3 does not match any compounds in the Select library and was an isotope peak of target 1 .

Example 1 demonstrates that, by using multiple is-CID mass spectra, we are able to correctly assign the reference acetyl fentanyl a greater classification index than the reference benzyl fentanyl. This is true using either of the considered spectral similarity scoring methods. While there are some use cases where knowing the mixture contains one or the other of the fentanyl analogs will be sufficient, the ILSA gives the analyst a more detailed profile of their mixture. For brevity, we will only discuss classification indices using FPIE with equal weighting between spectra as a measure of spectral similarity for the remaining examples. RevMF scores will be discussed again in the complete results presented in the Section 3.2. The impact of weighting on spectral similarity scores has yet to be explored in detail. Exploring weightings, in addition to other questions about spectral similarity scoring with a more diverse test set of data, would be appropriate future work. 

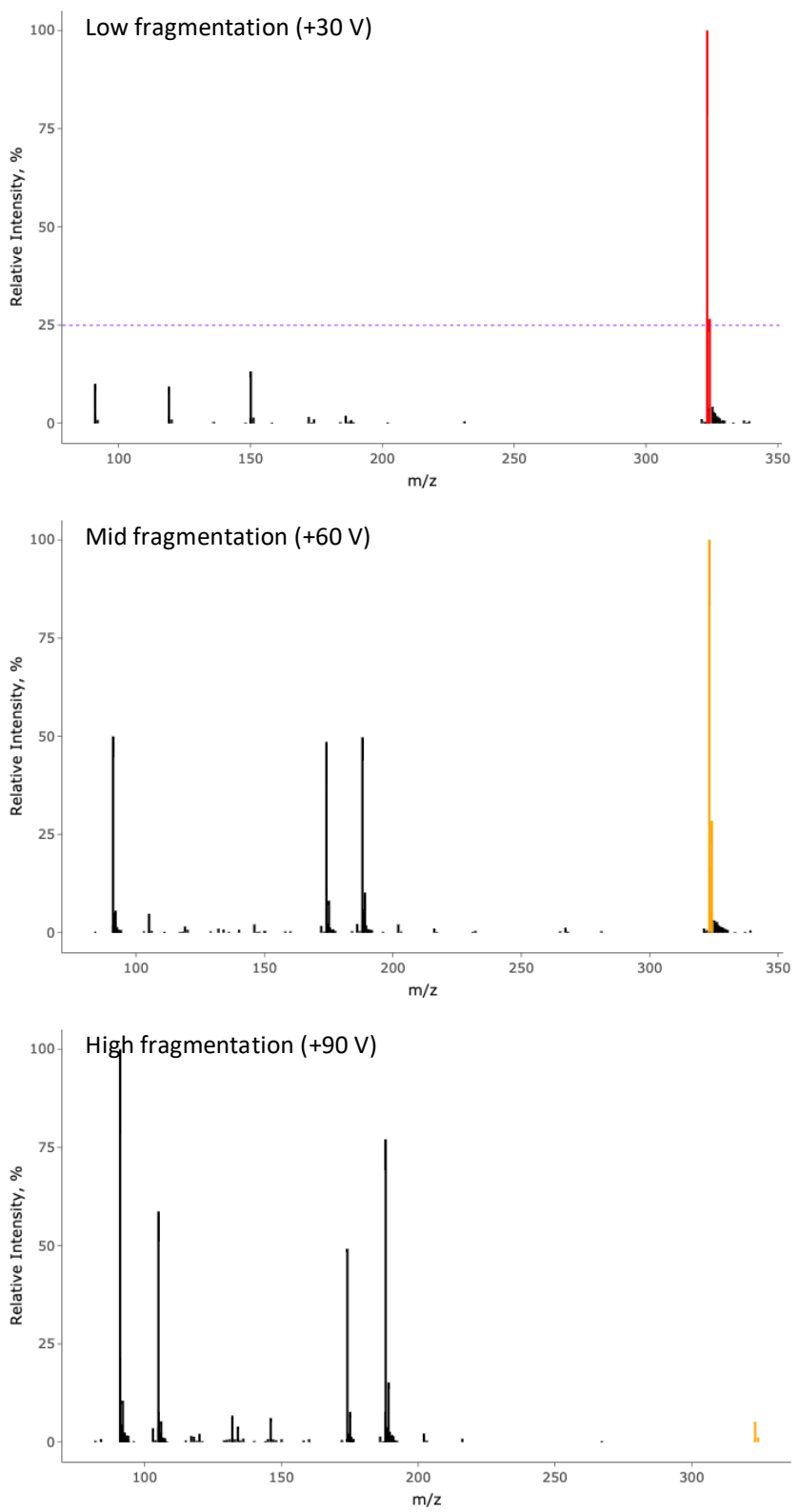

Figure 3: In-source collision-induced dissociation (is-CID) mass spectra of Mixture 8 (Table 1) measured using a JEOL AccuTOF (Peabody, MA, USA) mass spectrometer, with orifice 1 energy settings of $+30 \mathrm{~V},+60 \mathrm{~V}$ and $+90 \mathrm{~V}$. Potential targets with a relative intensity greater than $25 \%$ (i.e., with peak intensities above dashed horizontal line) in $+30 \mathrm{~V}$ spectrum are highlighted in red. The peaks corresponding to the target(s) identified in the low fragmentation spectrum are highlighted in orange in the higher fragmentation spectra. This figure is discussed in Example 2 of Section 3.1.

As a second example, consider Mixture 8 from Table 1 that includes benzyl fentanyl in addition to acetyl fentanyl and methamphetamine. The is-CID mass spectra are shown in Figure 3. Using a relative intensity threshold $\tau_{M P}=25 \%$, only two targets are identified. Similar to the previous example, target 1 , with $\mathrm{m} / \mathrm{z}$ 323.212 and relative intensity $100 \%$, matches the protonated molecule for acetyl fentanyl and benzyl fentanyl from the Select library. In this case, the computed classification indices of 0.951 and 0.942 for acetyl fentanyl and benzyl fentanyl, respectively, suggest (correctly) that both compounds are in the 
mixture. Target 2, with $\mathrm{m} / \mathrm{z} 324.215$ and relative intensity $26.6 \%$, does not match any compounds in the select library and is an isotope peak of target 1 . If we reduce the relative intensity threshold to $\tau_{R I}=13 \%$, we identify a third target, with $\mathrm{m} / z 150.129$ and relative intensity $13.1 \%$, that matches the protonated molecule of methamphetamine from the Select library with a classification index of 0.949 .

Example 2 demonstrates one of the operational challenges of using the ILSA (see Remark 1 from Section 2.1). By dropping our relatively intensity threshold we are able to identify all three components of the mixture. More discussion of the target identification stage of the algorithm is to follow the last example.

As a third and final example, consider Mixture 11 from Table 1 that includes all four of acetyl fentanyl, benzyl fentanyl, amphetamine and methamphetamine. The is-CID mass spectra are shown in Figure 4. In this example, we set the relative intensity threshold to $\tau_{M P}=9 \%$, identifying 5 possible target $m / z$ values. As with the other examples, target 1 , with $\mathrm{m} / z \quad 323.214$ and relative intensity $100 \%$, matches the protonated molecules for both acetyl fentanyl and benzyl fentanyl from the select library. In this case the classification indices of 0.934 and 0.930 for acetyl fentanyl and benzyl fentanyl, respectively, suggest (correctly) that both compounds are in the mixture. Target 2, with $m / z$ value of 324.217 Da and relative intensity of $26.8 \%$, does not match any compounds in the Select library and is an isotope peak of target 1 . Target 3, with $\mathrm{m} / z 91.056$ and relative intensity $12.5 \%$, and target 4 , with $\mathrm{m} / z 119.008$ and relative intensity $11.7 \%$ do not match any of the protonated molecules in the Select library. Target 5 , with $\mathrm{m} / \mathrm{z}$ 150.130 and relative intensity $9.9 \%$, matches the protonated molecule of methamphetamine with a classification index of 0.934 suggesting methamphetamine is in the mixture. If we drop the relative intensity threshold for target identifications down to $1 \%$, we will identify, as target 15 with $m / z 136.115$ and relative intensity $1.6 \%$, the protonated molecule of amphetamine with a classification index of 0.969 , suggesting amphetamine is in the mixture. Targets 6 to 14 were fragment ions that did not match any protonated molecules in the Select library.

Example 3 further illustrates the challenge of identifying targets by protonated molecules using a relative intensity threshold. This challenge is heightened as the protonated molecule for amphetamine is often the second or third most intense peak in its $+30 \mathrm{~V}$ spectrum (with the other prominent peaks observed $\mathrm{m} / \mathrm{z}$ 91.056 and 119.008). One work-around, as initially noted in Remark 1 from Section 2.1, is to search the library for spectra with base peaks that match the target $m / z$ rather than protonated molecules. Following such a strategy, target 3 in Example 3, with $\mathrm{m} / z 91.056$ and relative intensity of $12.5 \%$, matches the base peak of the $+30 \mathrm{~V}$ amphetamine spectrum contained in the select library. Accordingly, all four compounds would have been identified within the top 5 targets and with a relative intensity threshold of $11 \%$. Note that for reference compounds where the protonated molecule is the base peak in the $+30 \mathrm{~V}$ spectrum, with no mass calibration errors, targeting by protonated molecule or base peak will produce equivalent scores. 

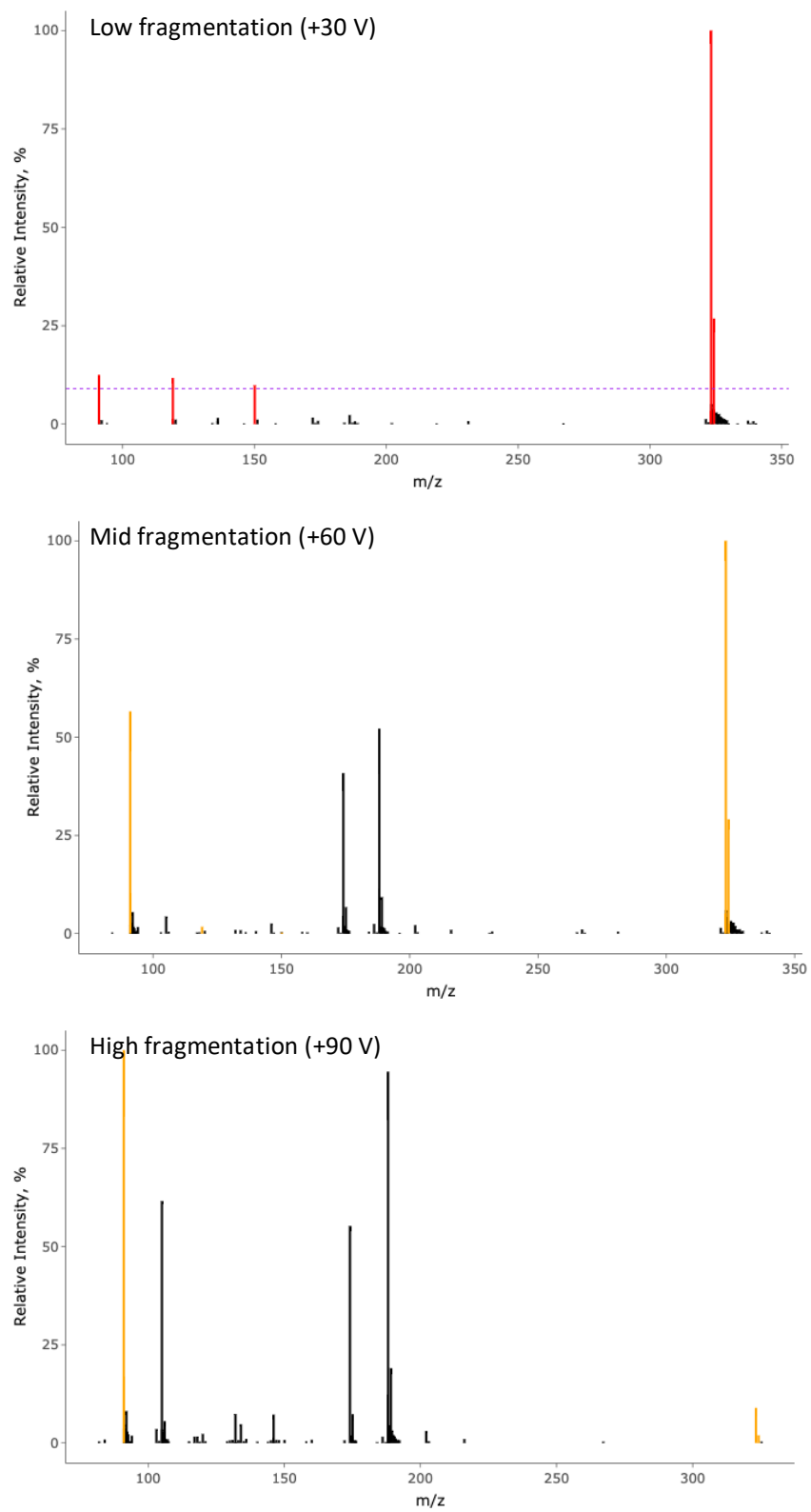

Figure 4: In-source collision-induced dissociation (is-CID) mass spectra of Mixture 11 (Table 1) measured using a JEOL AccuTOF (Peabody, MA, USA) mass spectrometer, with orifice 1 energy settings of $+30 \mathrm{~V},+60 \mathrm{~V}$ and $+90 \mathrm{~V}$. Potential targets with a relative intensity greater than 9\% (i.e., with peak intensities above dashed horizontal line) in $+30 \mathrm{~V}$ spectrum are highlighted in red. The peaks corresponding to the target(s) identified in the low fragmentation spectrum are highlighted in orange in the higher fragmentation spectra. This figure is discussed in Example 3 of Section 3.1. 


\subsection{Complete Results and Discussion}

The complete results of searching each of the 11 mixtures against both the select and NIST DART-MS Forensics 2020 library is detailed in Table 2. For all searches, the initial relative intensity threshold considered for identifying target molecules was set to $25 \%$, the tolerance window for $m / z$ errors $\left(\epsilon_{0}, \epsilon_{1}\right)$ was set to $\pm 0.005 \mathrm{~m} / \mathrm{z}$, and targets were matched to protonated molecules from the libraries. Threshold relative intensities were incrementally dropped by $1 \%$ until all expected compounds were identified for the purpose of demonstration.

Based on the notes in Remark 1 of Section 2.1 and Examples 2 and 3 in Section 3.1, it was clear that the target identification stage of the ILSA would struggle to detect a mixture component like amphetamine where the protonated molecule is often the second or third most prominent peak in the spectrum. The relative intensity threshold required to detect the protonated molecules for amphetamine was always less than $5 \%$, and as low as $1 \%$ in the three of the test mixtures. In two of the three test mixtures where we had to drop the relative intensity to $1 \%$, the ILSA found benzyl acryl fentanyl as a target, from the NIST library, for another low signal peak. The classification indices computed for benzyl acryl fentanyl were between 0.550 and 0.901 . We will return to the interpretation of classification indices later in this discussion but note that these indices should not be considered reliable for scenarios where the target is matched to a protonated molecule in the database and yet the target relative intensity is very low. We have mentioned the notion of assuming detected targets are base peaks, which in this set of tests would have ensured we did not explore targets with very low relative intensities. However, it is likely that even more sophisticated methods for target identification are to be discovered that better utilize the metadata available in the database and the multiple is-CID spectra available for the mixture. For example, can we make assumptions about the presence or absence of adducts? Or can we possibly look for common neutral losses to confirm whether an identified target is a protonated molecule?

There is a general trend worth noting about the scoring stage of the ILSA. Classification indices computed using FPIE as a spectral similarity score were always greater for correct matches from the select library than the NIST library. This is not at all surprising as the spectra in the select library were collected simultaneously with the mixture spectra. Accordingly, the mixture spectra and select library spectra are likely to have similar features and background ions that are captured through the FPIE computation. Similarly, the computed classification indices leveraging RevMFs as similarity scores were also mostly greater for matches from the select library than the NIST library, with the occasional exception. Interestingly, these exceptions were always observed for matches of either acetyl fentanyl or benzyl fentanyl (see mixtures 1, 4, 5, 7, 8, 10, 11). There are two reasons why RevMF-based scores would be higher for one library spectrum than another: (1) there are more peaks present in the spectrum to be matched by peaks in the mixture, and (2) the relative intensity values of peaks in the spectrum and the matching peaks in the mixture share a similar pattern. With either scoring method (FPIE or RevMF) there are problematic scenarios and edge-cases with less than desirable performance. Identifying and characterizing these cases is something we are presently exploring both mathematically and empirically. 
Table 2: Summarized results of searching 11 mixtures from Table 1 against select library of mixture component spectra collected at time of analysis and the general purpose NIST DART-MS Forensics Database. Targets not listed per search did not have any matches with either library by protonated molecule. Classification index $\theta_{i}^{F P I E}$ is computed using fraction of library peak intensity explained in mixture as a spectral similarity score, and $\theta_{i}^{R e v M F}$ is computed using the reverse match factor (both measures clarified in Appendix A). Note, since acetyl and benzyl fentanyl have the same protonated molecule, they are listed as matches for the same target. Bold-faced matches indicate compounds included in the mixtures.

\begin{tabular}{|c|c|c|c|c|c|c|c|c|}
\hline \multirow[b]{3}{*}{ Mixture } & \multirow[b]{3}{*}{$\tau_{R I}$} & \multirow[b]{3}{*}{ Target } & \multirow[b]{3}{*}{ m/z (Rel. Intensity) } & \multirow[b]{3}{*}{ Match } & \multicolumn{4}{|c|}{ Library } \\
\hline & & & & & \multicolumn{2}{|c|}{ Select } & \multicolumn{2}{|c|}{ NIST } \\
\hline & & & & & $\theta_{i}^{F P I E}$ & $\theta_{i}^{R e v M F}$ & $\theta_{i}^{F P I E}$ & $\theta_{i}^{R e v M F}$ \\
\hline \multirow{2}{*}{1} & \multirow{2}{*}{$25 \%$} & 1 of 2 & $323.212(100.0 \%)$ & Acetyl fentanyl & 0.950 & 0.938 & 0.919 & 0.993 \\
\hline & & & & Benzyl fentanyl & 0.947 & 0.924 & 0.931 & 0.877 \\
\hline \multirow{3}{*}{2} & \multirow{3}{*}{$5 \%$} & 1 of 5 & $323.213(100.0 \%)$ & Acetyl fentanyl & 0.980 & 0.996 & 0.921 & 0.979 \\
\hline & & & & Benzyl fentanyl & 0.635 & 0.645 & 0.538 & 0.645 \\
\hline & & 5 of 5 & $136.114(5.6 \%)$ & Amphetamine & 0.963 & 0.989 & 0.813 & 0.708 \\
\hline \multirow{4}{*}{3} & \multirow{4}{*}{$25 \%$} & 1 of 3 & $323.214(100.0 \%)$ & Acetyl fentanyl & 0.979 & 0.992 & 0.928 & 0.974 \\
\hline & & & & Benzyl fentanyl & 0.642 & 0.657 & 0.535 & 0.644 \\
\hline & & 2 of 3 & $150.130(26 \%)$ & Methamphetamine & 0.895 & 0.969 & 0.888 & 0.696 \\
\hline & & & & Phentermine & - & - & 0.825 & 0.691 \\
\hline \multirow{3}{*}{4} & \multirow{3}{*}{$3 \%$} & 1 of 6 & $323.214(100 \%)$ & Acetyl fentanyl & 0.479 & 0.591 & 0.537 & 0.669 \\
\hline & & & & Benzyl fentanyl & 0.970 & 0.969 & 0.934 & 0.979 \\
\hline & & 5 of 6 & $136.116(3.6 \%)$ & Amphetamine & 0.970 & 0.988 & 0.805 & 0.951 \\
\hline \multirow{4}{*}{5} & \multirow{4}{*}{$18 \%$} & 1 of 3 & $323.214(100.0 \%)$ & Acetyl fentanyl & 0.556 & 0.591 & 0.643 & 0.676 \\
\hline & & & & Benzyl fentanyl & 0.968 & 0.974 & 0.937 & 0.978 \\
\hline & & 3 of 3 & $150.130(18.4 \%)$ & Methamphetamine & 0.948 & 0.993 & 0.939 & 0.954 \\
\hline & & & & Phentermine & - & - & 0.794 & 0.915 \\
\hline \multirow{3}{*}{6} & \multirow{3}{*}{$10 \%$} & 3 of 4 & $150.130(85.2 \%)$ & Methamphetamine & 0.981 & 0.989 & 0.969 & 0.921 \\
\hline & & & & Phentermine & - & - & 0.776 & 0.880 \\
\hline & & 4 of 4 & $136.115(10.8 \%)$ & Amphetamine & 0.991 & 0.982 & $\mathbf{0 . 8 0 7}$ & 0.952 \\
\hline \multirow{3}{*}{7} & \multirow{3}{*}{$3 \%$} & 1 of 7 & $323.215(100.0 \%)$ & Acetyl fentanyl & 0.949 & 0.921 & 0.916 & 0.988 \\
\hline & & & & Benzyl fentanyl & 0.940 & 0.918 & 0.923 & 0.863 \\
\hline & & 7 of 7 & $136.115(3.4 \%)$ & Amphetamine & 0.989 & 0.991 & 0.810 & 0.897 \\
\hline & & 1 of 3 & $323.212(100.0 \%)$ & Acetyl fentanyl & 0.951 & 0.892 & 0.919 & 0.994 \\
\hline 8 & & & & Benzyl fentanyl & 0.942 & 0.937 & 0.928 & 0.883 \\
\hline 8 & $13 \%$ & 3 of 3 & $150.129(13.2 \%)$ & Methamphetamine & 0.949 & 0.995 & 0.945 & 0.910 \\
\hline & & & & Phentermine & - & - & 0.826 & 0.886 \\
\hline & & 1 of 24 & $323.214(100.0 \%)$ & Acetyl fentanyl & 0.989 & 0.987 & 0.921 & 0.977 \\
\hline & & & & Benzyl fentanyl & 0.656 & 0.688 & 0.538 & 0.645 \\
\hline 9 & $1 \%$ & 5 of 24 & $150.129(18.8 \%)$ & Methamphetamine & 0.949 & 0.981 & 0.930 & 0.733 \\
\hline & & & & Phentermine & - & - & 0.854 & 0.721 \\
\hline & & 14 of 24 & $136.115(1.9 \%)$ & Amphetamine & 0.976 & 0.980 & 0.813 & 0.775 \\
\hline & & 1 of 23 & $323.213(100.0 \%)$ & Acetyl fentanyl & 0.447 & 0.591 & 0.536 & 0.671 \\
\hline & & & & Benzyl fentanyl & 0.953 & 0.967 & 0.940 & 0.982 \\
\hline 10 & & 4 of 23 & $150.129(15.0 \%)$ & Methamphetamine & 0.956 & 0.990 & 0.947 & 0.928 \\
\hline 10 & $1 \%$ & & & Phentermine & - & - & 0.778 & 0.890 \\
\hline & & 13 of 23 & $321.198(2.1 \%)$ & Benzyl Acryl fentanyl & - & - & 0.901 & 0.626 \\
\hline & & 19 of 23 & $136.114(1.4 \%)$ & Amphetamine & 0.977 & 0.981 & 0.802 & 0.951 \\
\hline & & 1 of 22 & $323.214(100.0 \%)$ & Acetyl fentanyl & 0.934 & 0.909 & 0.916 & 0.989 \\
\hline & & & & Benzyl fentanyl & 0.930 & 0.924 & 0.926 & 0.890 \\
\hline 11 & 10 & 5 of 22 & $150.130(9.9 \%)$ & Methamphetamine & 0.934 & 0.983 & 0.931 & 0.862 \\
\hline 11 & $1 \%$ & & & Phentermine & - & - & 0.829 & 0.838 \\
\hline & & 15 of 22 & $136.115(1.6 \%)$ & Amphetamine & 0.969 & 0.983 & 0.809 & 0.910 \\
\hline & & 19 of 22 & $321.198(1.3 \%)$ & Benzyl Acryl fentanyl & - & - & 0.886 & 0.550 \\
\hline
\end{tabular}


In addition to determining the range of utility for each of the considered similarity scores, it may also be worth exploring the impact of weighting on computed parameters, considering other existing similarity scores, developing novel metrics for similarity, or computing agglomerative values that include multiple similarity metrics. In the results presented in this manuscript, the computed classification indices were disaggregated by type of similarity score (FPIE vs RevMF) for exploratory and analytical reasons. However, it may be useful to employ both classification indices when making decisions, either manually or through an automated classification system.

One of the least satisfying aspects of the results presented in this manuscript is the unclear distinction between "good" and "bad" classification indices based on the limited test sets of mixtures we considered. As we noted previously in our discussion of benzyl acryl fentanyl, classifier indices should be viewed with caution when computed for targets identified with very low relative intensities. But even for prominent targets some care must be taken when interpreting classification indices. In mixtures that contained one or both of acetyl fentanyl and benzyl fentanyl, the average classification index of the true compound in the mixture using both libraries and both scoring schemes was $0.945(n=56)^{3}$, and the average classification index of the compound not in the mixture using both libraries and both scoring schemes was $0.602(\mathrm{n}=24)$. These substantial differences in classification indices suggest we can determine with confidence which fentanyl analogs are in the mixture. In contrast, for mixtures where both methamphetamine and phentermine were identified as a target using the general NIST library the average classification index using either scoring scheme was $0.897(\mathrm{n}=14)$ for methamphetamine, the compound in the mixture, and $0.822(\mathrm{n}=14)$ for phentermine, the compound not in the mixture. Further, if we used only the RevMF scoring scheme to compute classification indices, the discrepancy was even more muted, with the average classification index being $0.858(\mathrm{n}=7)$ for methamphetamine and $0.832(\mathrm{n}=7)$ for phentermine. Rather than select a single classification threshold value for all decision making, it may be advantageous to set several decision threshold indices for classes of compounds to try to best characterize mixture components. Determining these thresholds will require a well-designed set of training and testing data that includes a large number of annotated mixtures.

With the limited number of mixture components, test mixture compositions, and experimental conditions considered in this manuscript, it is difficult to make universal claims about how well the ILSA will perform in practical situations. Nevertheless, the results of this manuscript suggest that this multi-stage inverted approach to targeting and scoring spectra, especially with further improvements in the areas outlined in this manuscript, has promise as an effective method for identifying mixture components using DART-MS.

\section{Disclaimer}

Official contribution of the National Institute of Standards and Technology (NIST); not subject to copyright in the United States. Certain commercial products are identified in order to adequately specify the procedure; this does not imply endorsement or recommendation by NIST, nor does it imply that such products are necessarily the best available for the purpose.

\footnotetext{
${ }^{3}$ There are 56 classification values listed in Table 1 for acetyl fentanyl and benzyl fentanyl when they are present in the mixture. The $n$ values reported in the subsequent discussion were obtained in the same manner for the different scenarios described.
} 


\section{References}

1. Cody, R.B., Laramée, J.A., Durst, H.D.: Versatile New Ion Source for the Analysis of Materials in Open Air under Ambient Conditions. Anal. Chem. 77, 2297-2302 (2005). https://doi.org/10.1021/ac050162j

2. Gross, J.H.: Direct analysis in real time - a critical review on DART-MS. Anal Bioanal Chem. 406, 63-80 (2014). https://doi.org/10.1007/s00216-013-7316-0

3. Sisco, E., Forbes, T.P.: Forensic applications of DART-MS: A review of recent literature. Forensic Chemistry. 22, 100294 (2021). https://doi.org/10.1016/j.forc.2020.100294

4. Stein, S.: Mass Spectral Reference Libraries: An Ever-Expanding Resource for Chemical Identification. Anal. Chem. 84, 7274-7282 (2012). https://doi.org/10.1021/ac301205z

5. Sisco, E., Moorthy, A.S., Watt, L.M.: Creation and Release of an Updated NIST DART-MS Forensics Database. J. Am. Soc. Mass Spectrom. 32, 685-689 (2021). https://doi.org/10.1021/jasms.0c00416

6. Sisco, E., Moorthy, A.S.: NIST DART-MS Forensics Database 2020, https://data.nist.gov/od/id/mds2-2313, (2020)

7. Stein, S.E.: NIST MS Search v.2.3.

8. Moorthy, A.S., Wallace, W.E., Kearsley, A.J., Tchekhovskoi, D.V., Stein, S.E.: Combining Fragment-Ion and Neutral-Loss Matching during Mass Spectral Library Searching: A New General Purpose Algorithm Applicable to Illicit Drug Identification, https://pubs.acs.org/doi/pdf/10.1021/acs.analchem.7b03320

9. Burke, M.C., Mirokhin, Y.A., Tchekhovskoi, D.V., Markey, S.P., Heidbrink Thompson, J., Larkin, C., Stein, S.E.: The Hybrid Search: A Mass Spectral Library Search Method for Discovery of Modifications in Proteomics. J. Proteome Res. 16, 1924-1935 (2017). https://doi.org/10.1021/acs.jproteome.6b00988

10. Cooper, B.T., Yan, X., Simón-Manso, Y., Tchekhovskoi, D.V., Mirokhin, Y.A., Stein, S.E.: Hybrid Search: A Method for Identifying Metabolites Absent from Tandem Mass Spectrometry Libraries. Anal. Chem. 91, 13924-13932 (2019). https://doi.org/10.1021/acs.analchem.9b03415

11. Stein, S.E., Scott, D.R.: Optimization and testing of mass spectral library search algorithms for compound identification. J Am Soc Mass Spectrom. 5, 859-866 (1994). https://doi.org/10.1016/1044-0305(94)87009-8

12. Moorthy, A.S., Kearsley, A.J.: (To appear) Pattern similarity measures applied to mass spectra. In: Progress in Industrial Mathematics: Success stories. Springer International Publishing (2021)

13. R Core Team: R: A language and environment for statistical computing. R Foundation for Statistical Computing, Vienna, Austria. URL https://www.R-project.org/. (2019)

14. Chang, W., Cheng, J., Allaire, J., Xie, Y., McPherson, J.: shiny: Web Application Framework for R. (2020) 


\section{Appendix A: Example Calculations.}

In this appendix, we explicitly compute similarity scores of importance to this manuscript with simplified example data not representing any real compounds or mixtures. Our intent is to provide detailed steps that were outside of the scope of the main text.

Recall from the manuscript that we denote the mass spectrum of a library compound $i$ measured with fragmentation condition $E$ as $l_{i}^{E}$, and the mass spectrum of a mixture measured under the same fragmentation condition as $x_{M}^{E}$.

\section{Example 1: Integer resolution query and library mass spectra}

Consider the following integer-resolution library and mixture mass spectra,

$$
\begin{gathered}
l_{i}^{E}=\{(10,5),(15,30),(25,100)\}, \\
x_{M}^{E}=\{(5,20),(9,2),(12,25),(15,17),(25,100),(28,20)\},
\end{gathered}
$$

where each spectrum can be interpreted as a set of coordinates representing peak $m / z$ values and relative intensities (e.g., the library spectrum has a peak at $m / z 10$ with relative intensity 5 ).

Fraction of library peak intensity explained (FPIE): Two of the three peaks in $l_{i}^{E}$ have "matching" peaks (matched by $m / z$ value) in $x_{M}^{E}$. The total relative intensity of the peaks in $l_{i}^{E}$ with matching peaks in $x_{M}^{E}$ is $30+100=130$. The total relative intensity of peaks in $l_{i}^{E}$ is 135 . The fraction of library peak intensity explained by matching peaks in the mixture is $130 / 135 \approx 0.963$.

Reverse Match Factor (RevMF): A vector of the relative intensities in spectrum $l_{i}^{E}$ can be constructed as $\lambda=[5,30,100]$. A vector of the relative intensities in spectrum $x_{M}^{E}$ paired with $m / z$ values from $l_{i}^{E}$ can be constructed as $\chi=[0,17,100]$. Note that spectrum $x_{M}^{E}$ does not contain a peak at $\mathrm{m} / z 10$ hence the first element of $\chi$ is 0 . The cosine similarity between vectors $\lambda$ and $\chi$ is defined

$$
\cos \operatorname{sim}(\lambda, \chi)=\frac{\sum_{i}\left(\lambda_{i} \times \chi_{i}\right)}{\sqrt{\sum_{i} \lambda_{i}^{2}} \times \sqrt{\sum_{i} \chi_{i}^{2}}}
$$

Explicitly computed, the cosine similarity between vectors $\lambda$ and $\chi$ is

$$
\cos \operatorname{sim}(\lambda, \chi)=\frac{(5 \times 0)+(30 \times 17)+(100 \times 100)}{\sqrt{\left(5^{2}+30^{2}+100^{2}\right)} \times \sqrt{\left(0^{2}+17^{2}+100^{2}\right)}} \approx \frac{10510}{10602} \approx 0.991 .
$$

This similarity measure is traditionally scaled by 100 or 1000 in certain software packages and termed a reverse match factor. In this manuscript we leave the value unscaled. 


\section{Example 2: High resolution query and library mass spectra}

Consider the following high-resolution library and mixture mass spectra,

$$
\begin{gathered}
l_{i}^{E}=\{(10.008,5),(15.115,30),(25.002,100)\} \\
x_{M}^{E}=\{(5.004,20),(9.293,2),(12.003,25),(15.117,17),(25.004,35),(25.006,100),(28,20)\} .
\end{gathered}
$$

Let the expected mass spectral resolution for $m / z$ values to be $\epsilon_{1}= \pm 0.005$.

FPIE: Two of the three peaks in $l_{i}^{E}$ have "matching" peaks (matched by $m / z$ values within $\epsilon_{1}$ ) in $x_{M}^{E}$. Note that two peaks in $x_{M}^{E}$ are within $\epsilon_{1}$ of the $m / z 25.002$ peak in $l_{i}^{E}$. Because the FPIE is only concerned with the relative intensities of peaks in the library spectrum, calculations can proceed with no issue. The total relative intensity of the peaks in $l_{i}^{E}$ with matching peaks in $x_{M}^{E}$ is $30+100=130$. The total relative intensity of peaks in $l_{i}^{E}$ is 135 . The fraction of library peak intensity explained by matching peaks in the mixture is $130 / 135 \approx 0.963$.

RevMF: A vector of the relative intensities in spectrum $l_{i}^{E}$ can be constructed as $\lambda=[5,30,100]$. A vector of the relative intensities in spectrum $x_{M}^{E}$ with the closest $m / z$ values from $l_{i}^{E}$ can be constructed as $\chi=$ $[0,17,35]$. Note that two peaks in $x_{M}^{E}$ are within \pm 0.005 of the $m / z 25.002$ peak in $l_{i}^{E}$. The relative intensity from the peak with $m / z$ value of 25.004 is used in the construction of $\chi$ as $|25.002-25.004|<$ $|25.002-25.006|$. It is worth noting, however, that this method of determining which peak intensity should be considered in the scoring is an area for further investigation and may yield insights.

Explicitly computed, the cosine similarity between vectors $\lambda$ and $\chi$ is

$$
\cos \operatorname{sim}(\lambda, \chi)=\frac{(5 \times 0)+(30 \times 17)+(100 \times 35)}{\sqrt{\left(5^{2}+30^{2}+100^{2}\right)} \times \sqrt{\left(0^{2}+17^{2}+35^{2}\right)}} \approx \frac{4010}{4067} \approx 0.986
$$

\section{Example 3: "Mixed" resolution query and library mass spectra}

Consider the following integer-resolution library mass spectrum and high-resolution mixture mass spectrum,

$$
\begin{gathered}
l_{i}^{E}=\{(10,5),(15,30),(25,100)\} \\
x_{M}^{E}=\{(5.004,20),(9.293,2),(12.003,25),(15.117,17),(25.004,35),(25.006,65),(28,20)\} .
\end{gathered}
$$

In a scenario where the query and library mass spectra are "mixed" resolution, the high-resolution mass spectrum can be approximated by an integer resolution mass spectrum where $m / z$ values are rounded and peaks with the same $m / z$ are consolidated by summing intensity. Accordingly, the "coarse" approximation of $x_{M}^{E}$ is $\tilde{x}_{M}^{E}=\{(5,20),(9,2),(12,25),(15,17),(25,100),(28,20)\}$ and the scoring proceeds as in Example 1 . 